\title{
A novel assay for neutrophil clustering activity of human sera: relation to disease activity and neutropenia in systemic lupus erythematosus
}

\author{
H Jonsson, G Sturfelt
}

\begin{abstract}
A simple and reproducible method for the measurement of serum neutrophil clustering activity was developed. High clustering activity was found in 19/30 patients with active systemic lupus erythematosus (SLE), and $14 / 20$ of those with severe disease flares. In contrast, 0/10 patients with quiescent SLE and $2 / 20$ patients with rheumatoid arthritis had high neutrophil clustering activity. Particularly high clustering activity was found in patients with SLE with lupus glomerulonephritis and in certain patients with central nervous system disease. An inverse correlation was found between neutrophil clustering activity and peripheral blood neutrophil count in patients with SLE not treated with glucocorticoids, and clustering activity was high in all patients with low neutrophil counts in this group. A moderate correlation was found between neutrophil clustering activity and Clq binding circulating immune complexes. Non-steroidal anti-inflammatory drugs and glucocorticoids had little direct effect on neutrophil clustering activity.
\end{abstract}

The intravascular aggregation of neutrophils has been implicated in the pathogenesis of several diseases, such as adult respiratory distress syndrome ${ }^{1}$ haemodialysis induced respiratory distress, ${ }^{2}$ and neutropenia associated with cardiopulmonary bypass. ${ }^{3}$ Neutrophil aggregation causes vascular injury in animal models in vivo. ${ }^{4} 5$

The standard method for measuring neutrophil aggregation, which involves the use of a platelet aggregometer, is adequate for studying the effects of purified complement components and $N$-formyl-methionyl-leucyl-phenylalanine. ${ }^{26}$ This assay has been found to be insensitive, however, for measuring serum neutrophil aggregating activity, ${ }^{7}$ unless the aggregation response is augmented by cytochalasin $\mathbf{B} .^{8}$

The aim of this study was to develop a simple, reproducible assay for the effect of human sera on neutrophil aggregation, and to use this assay for studies of neutrophil behaviour in rheumatic disorders. As previously, ${ }^{9}$ we chose the term 'clustering' to describe microscopic cellular aggregates.

\section{Materials and methods} GRANULOCYTE PREPARATION

Heparinised venous blood from healthy members of the clinical staff was centrifuged at $200 \mathrm{~g}$ for 10 minutes. The pellet was kept and the supernatant centrifuged again at $1000 \mathrm{~g}$. This pellet containing platelets was discarded and the first pellet resuspended in the supernatant. This was subsequently allowed to form a sediment for 45 minutes at room temperature with an equal volume of $2 \%$ dextran T-500, and the leucocyte rich supernatant was withdrawn. This was layered on top of an Isopaque-Ficoll mixture and centrifuged at $1350 \mathrm{~g}$ for 15 minutes. The granulocyte pellet was resuspended in the original serum-dextran mixture. After lysis of erythrocytes with $0.87 \%$ ammonium chloride and centrifugation the granulocytes were washed twice and resuspended in Hanks's balanced salt solution, containing $0.5 \%$ human serum albumin, until use. This produced a granulocyte preparation that was $>99 \%$ free of mononuclear cells and platelets.

\section{CLUSTERING ASSAY}

Serum $(40 \mu \mathrm{l})$ was layered on an object glass at $37^{\circ} \mathrm{C}$ and incubated for 10 minutes. A $10 \mu l$ aliquot of granulocyte suspension (containing $10 \times 10^{6}$ granulocytes/ml unless specified) was then added to the centre of the serum drop and incubated at room temperature, whereafter a cover slip was added and sealed with nail polish. Maximum clustering was reached after incubation for one minute, and no evidence of resolving cell clusters was seen up to five minutes. An incubation time of one minute was therefore chosen and used in all experiments. This gave a stable microscopic sample not changing for at least four hours, during which counting was done.

Single and clustered granulocytes in a square area around the centre of the drop area were counted (fig 1), and the percentage of clustered cells after counting 200 single cells was calculated. All experiments were performed in duplicate and the results represent the mean value.

The percentage of cells clustered in the presence of the pooled normal serum (normal control) and the same pool activated with heat aggregated IgG were chosen as negative and positive controls. Neutrophil clustering activity units based on these controls were expressed on a linear arbitrary scale of 0 (pooled normal serum) to 1 (serum activated with aggregated IgG), and calculated by the formula:

\section{Neutrophil clustering activity $=$ ((\% clustered cells) $-($ normal control $))$ \\ $((\%$ clustering in IgG activated serum $)-($ normal control $))$}

Negative neutrophil clustering activity values thus denote less clustering than that observed in 


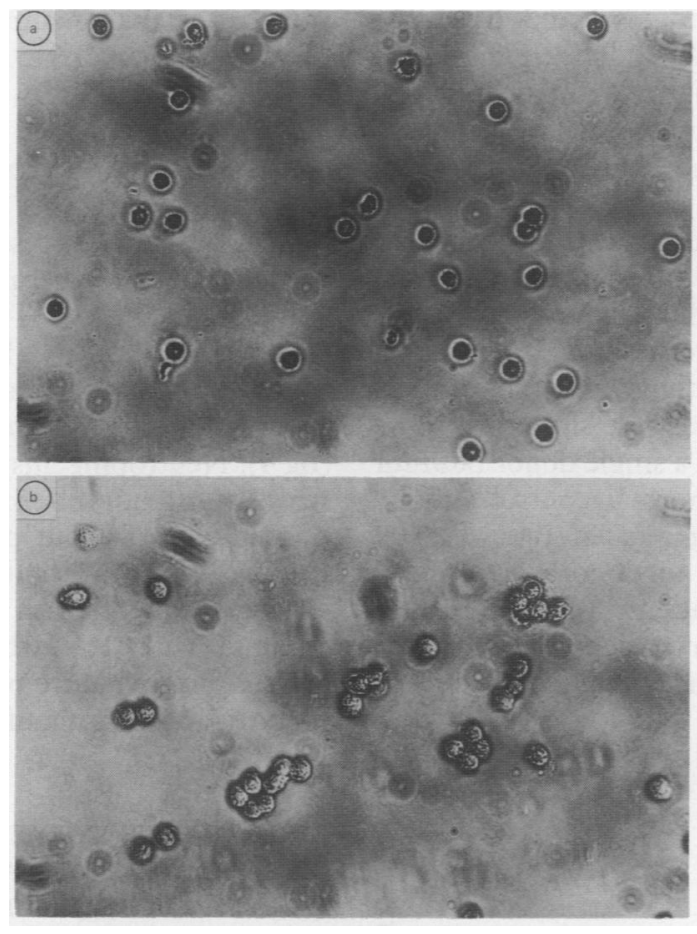

Figure 1: Neutrophilclustering in the presence of $(a)$ normal serum pool; $(b)$ serum from a patient with active systemic lupus erythematosus.

the normal serum pool, whereas serum samples with neutrophil clustering activity of more than 1 cause more clustering than the pool activated with aggregated IgG.

\section{PATIENTS}

All patients were attending our clinic. We studied 40 patients with systemic lupus erythematosus (SLE) all fulfilling at least four American Rheumatism Association criteria for the diagnosis of this disease. ${ }^{10}$ Disease activity was assessed on the basis of clinical examination and routine laboratory tests. In short, exacerbations demanding treatment in hospital or high prednisolone doses ( $\geqslant 20 \mathrm{mg} /$ day), or both, were considered major, whereas exacerbations that could safely be managed with other measures were termed minor. ${ }^{11}$ With this grading 20 patients with SLE had major flares, 10 had minor flares, and 10 had stable quiescent disease. None had evidence of intercurrent infection.

We also studied 20 patients with rheumatoid arthritis, who were consecutive outpatients fulfilling at least four American Rheumatism Association criteria for the diagnosis of this disease. ${ }^{12}$ Most patients had moderately active joint disease (Ritchie's index: median $7 \cdot 5$, range 0-22; erythrocyte sedimentation rate: median $24 \mathrm{~mm} / \mathrm{h}$, range $3-100$; $\mathrm{C}$ reactive protein: median $33 \mathrm{mg} / \mathrm{l}$, range $<12-180$ ). No patient had evidence of Felty's syndrome.

\section{SERUM SAMPLING}

Clotting was allowed to proceed for one hour at room temperature followed by one hour at $4^{\circ} \mathrm{C}$. Subsequently the samples were stored in small aliquots at $-70^{\circ} \mathrm{C}$ until use. Serum samples from patients with SLE were sampled at the time of maximum disease activity, before treatment was started.

Normal serum samples were acquired from 20 healthy members of the hospital staff and pooled for use as a negative control in all experiments.

Part of the normal serum pool was activated by IgG (Kabi Chemicals) dissolved in isotonic $\mathrm{NaCl}$ in a concentration of $10 \mathrm{mg} / \mathrm{ml}$, aggregated by warming to $63^{\circ} \mathrm{C}$ for 30 minutes, and subsequently centrifuged at $2000 \mathrm{~g}$ for 30 minutes. The supernatant was incubated for one hour at $37^{\circ} \mathrm{C}$ with the normal serum pool in the proportions $1: 10$, stored at $-70^{\circ} \mathrm{C}$ in small aliquots, and freshly thawed for each experiment.

\section{CIRCULATING IMMUNE COMPLEXES}

Circulating immune complexes were measured by a fluid phase $\mathrm{Clq}$ binding assay, according to Zubler et al. ${ }^{13}$

\section{STATISTICS}

Analysis of correlation was by the Spearman rank correlation test $\left(r_{s}\right)$, and differences between groups were analysed with the Mann-Whitney U test. The standard deviation (SD) of differences obtained on duplicate measurements was calculated to assess the random error of the method.

\section{Results}

Neutrophil cell clustering in the normal serum pool varied from $6 \%$ to $27 \%$ clustered cells, and in the serum pool activated by heat aggregated IgG from $31 \%$ to $55 \%$. These responses were linearly related, and the difference between the responses in normal and activated serum varied from $23 \%$ to $34 \%$. Figure 2 shows the effect of

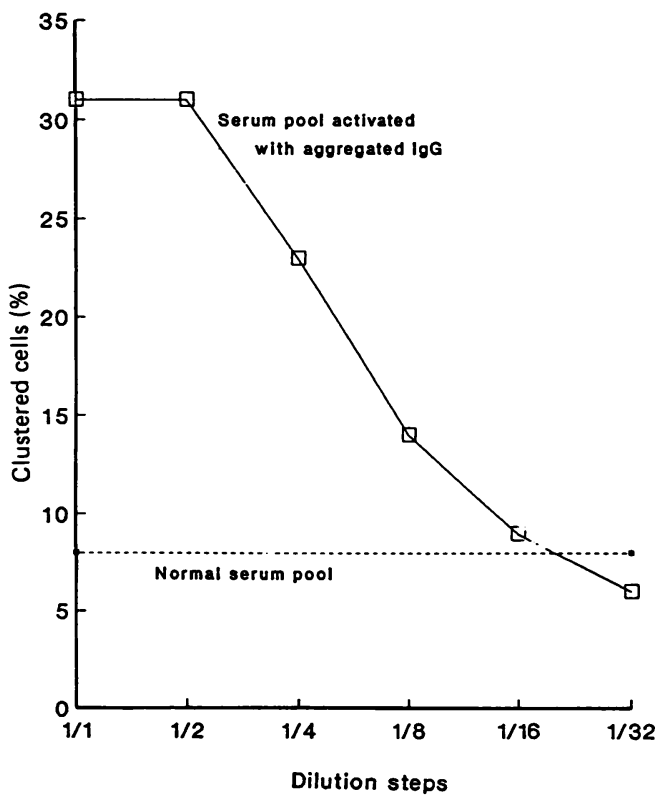

Figure 2: Percentage of neutrophil clustering caused by serum activated with aggregated IgG. The dilutions were done in the normal serum pool. 
dilutions of the activated pool and fig 3 the varying cell concentrations. The highest cell concentration was associated with an increased number of large cell aggregates, while a concentration of $10 \times 10^{6}$ allowed easy counting of cell aggregates in the centre of the sample. Reproducibility of the method, as expressed by the standard deviation of the differences in duplicate measurements in the 80 human sera (fig 4), was $6 \%$ clustered cells. Inter-donor variations in neutrophil clustering activity with neutrophils from six different donors were similar to those observed with cells from a single donor both for clustering induced by normal control and by serum activated with aggregated IgG. To test day to day, and donor to donor variation 12 serum samples from patients with SLE were analysed on two separate occasions. The standard deviation of the differences in the two experi-

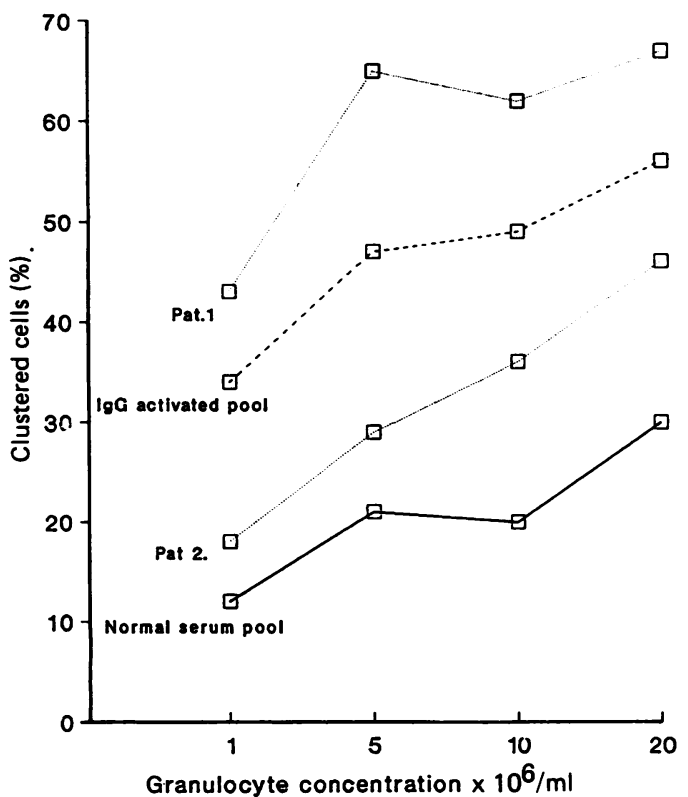

Figure 3: The effect of cell concentrations on the percentage of clustered neutrophils. The interval between the clustering in serum activated by aggregated IgG and non-activated serum is stable. Two serum samples from patients with systemic lupus erythematosus were included for comparison.

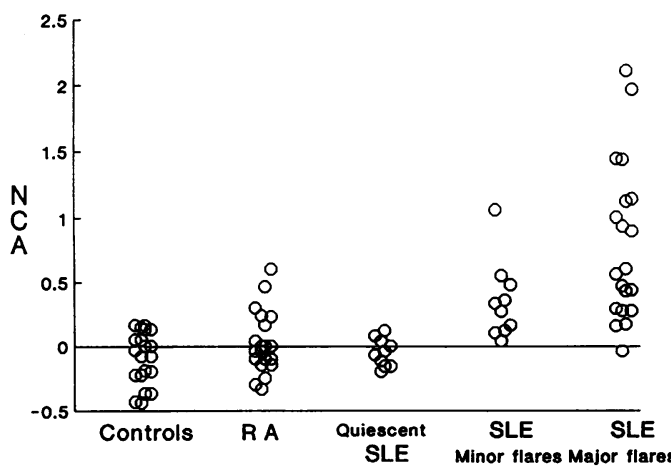

Figure 4: Neutrophil clustering activity (NCA) in control and patient serum samples. Serum samples from patients with active systemic lupus erythematosus ( $S E E)(n=30)$ had significantly higher neutrophil clustering activity than samples from normal controls $(n=20)$, and patients with rheumatoid arthritis $(R A)(n=20)$, or from patients with quiescent SLE ( $n=10)$ (Mann-Whimey $p<0.001$ ). Major SLE flares $(n=20)$ were associated with higher neutrophil clustering activity than were minor flares $(n=10)(p<0 \cdot 05)$. ments was 0.24 neutrophil clustering activity units and $r_{s}$ was 0.91 .

NEUTROPHIL CLUSTERING ACTIVITY IN CONTROL AND PATIENT SERUM SAMPLES

Normal range, expressed as mean (2 SD) of the normal control sera, was -0.5 to 0.32 (mean -0.09 , SD 0.205). Two of 20 patients with rheumatoid arthritis (RA), 5/10 patients with SLE with minor flares, and 14/20 with major flares had high neutrophil clustering activity (fig 4). Neutrophil clustering activity in patients with RA did not differ significantly from that in normal controls, but patients with active SLE had high clustering both compared with controls and with patients with $\mathrm{RA}(\mathrm{p}<0.001)$. The two patients with $R A$ who had high clustering activity had evidence of active joint disease but no extra-articular manifestations or neutropenia.

RELATION BETWEEN NEUTROPHIL CLUSTERING ACTIVITY AND DISEASE MANIFESTATIONS IN ACTIVE SLE

Patients with major manifestations of disease had higher neutrophil clustering activity than those with minor manifestations only $(p<0.05)$, and the patients with the highest clustering activity had severe flares. Patients with active nephritis $(n=10)$ had high neutrophil clustering activity (median 1.06, range $0 \cdot 16-2 \cdot 1$ ) compared with those with active disease without nephritis $(n=20)$ (median 0.31 , range -0.04 to 1.43 ; $p<0.01)$. No significant differences were found between patients with active SLE with and without central nervous system (CNS) disease $(n=9)$ or serositis $(n=12)$. Nor was there any difference in patients with active disease taking glucocorticoids $(n=14)$ or non-steroidal antiinflammatory drugs $(n=10)$ compared with the remainder of the patients with active disease.

RELATION TO LEUCOPENIA

Patients with total leucocyte count of $<4 \times 10^{9} / 1$

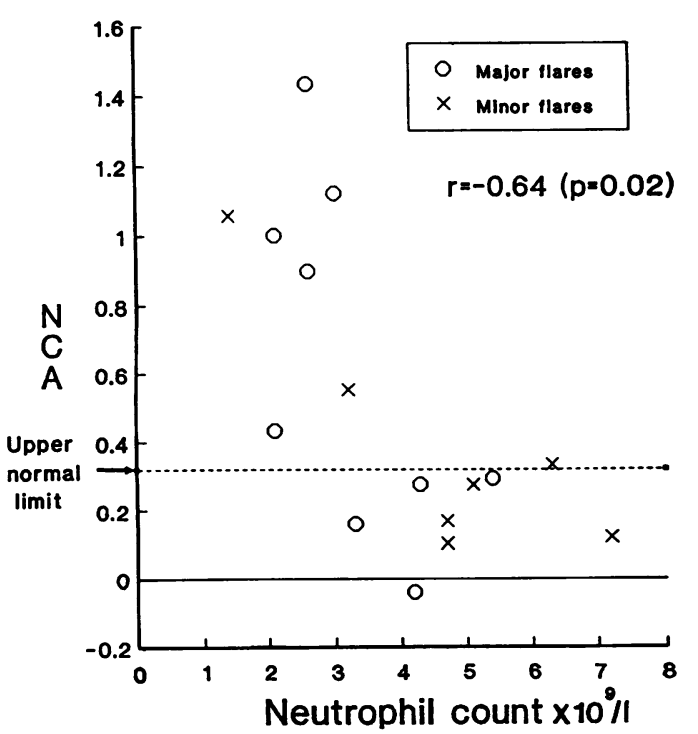

Figure 5: Neutrophil clustering activity (NCA) related to neutrophil count in patients with systemic lupus erythematosus ( $n=16)$ not treated with glucocorticoids at time of sampling.

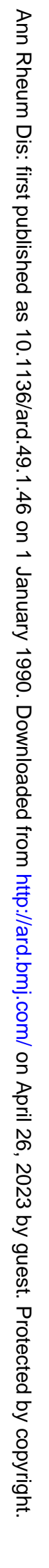


$(n=11)$ had significantly higher neutrophil clustering activity (median 0.9 , range 0.43 to $2 \cdot 1)$ than patients with normal leucocyte counts (median 0.28 , range -0.04 to $1.44 ; \mathrm{p}<0.01$ ). A similar but less marked difference was found in patients with a neutrophil count of $<3 \times 10^{9} / 1$ $(\mathrm{n}=11$, neutrophil clustering activity median 0.9 , range 0.43 to $2 \cdot 1$ ) than in patients with a greater number of neutrophils (median $0 \cdot 28$, range -0.04 to $1.96 ; p<0.01$ ). Lymphocyte and platelet counts were not related to neutrophil clustering activity in these patients.

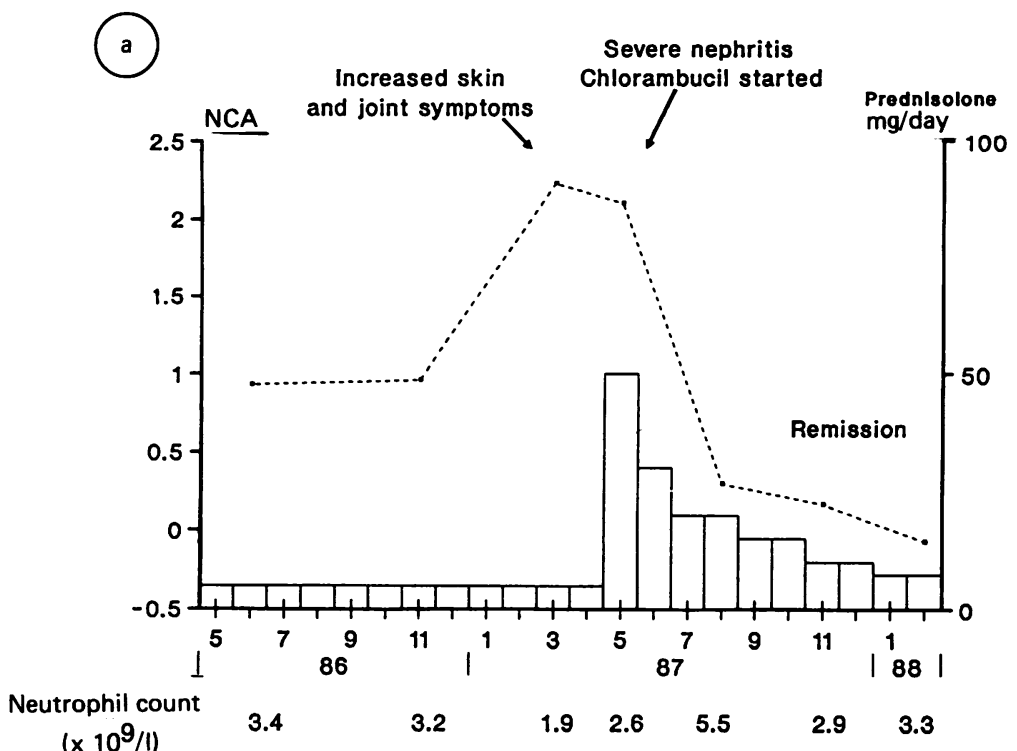
$\left(\times 10^{9} / 1\right)$
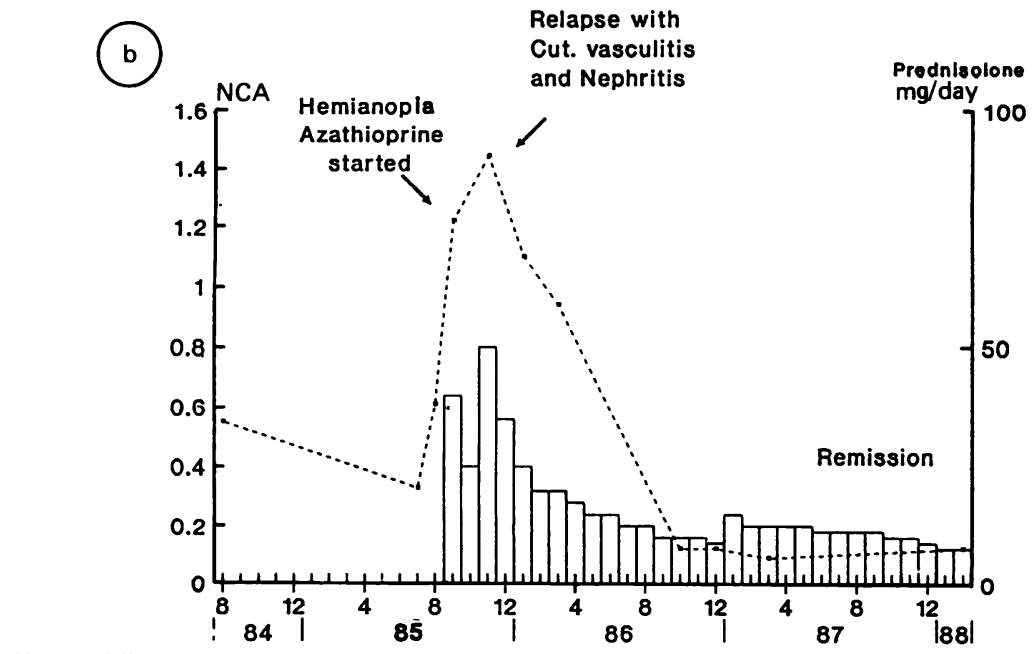

and Nephritis

Neutrophil count

$\left(\times 10^{9} / 1\right)$

2.4

3.93 .26 .8

2.9

3.1

Figure 6: Sequential neutrophil clustering activity (NCA) measurement and its relation to disease activity and immunosuppressive treatment. Neutrophil clustering activity is represented by the broken line and prednisolone dose by staples. (a) A 58 year old woman with systemic lupus erythematosus of 30 years' duration. She had an exacerbation of glomerulonephritis in 1983 and was treated with glucocorticoids and azathioprine. In 1986 her renal function was stable, but the patient had episodes of skin and joint symptoms. In the spring of 1987 she developed a severe flare with profuse proteinuria and decreased renal filtration, together with fever, leucopenia, thoracic pain, and central nervous system involvement with' lightning flashes'. She responded well to glucocorticoids and chlorambucil and is now completely asymptomatic. (b) This 36 year old woman had symmetric arthritis at the time of her first visit, but was found to have positive antinuclear antibodies, anti-dsDNA, and anti-Sm. She continued to have monosystemic disease and was treated with non-steroidal anti-inflammatory drugs. During the summer of 1985 the arthritis got worse and in September 1985 she presented with hemianopia and pathological urine casts together with a lymphopenia of $0 \cdot 3 \times 10^{9} / l$. Treatment was started with glucocorticoids and azathioprine and disease manifestations disappeared. Seven weeks later she had a severe relapse with large cutaneous vascular ulcers and recurrence of urine casts. She has subsequently improved and azathioprine was exchanged for chloroquine in fune 1987.
When all patients with SLE were included no correlation was found between neutrophil clustering activity and total leucocyte, neutrophil, or lymphocyte count, but when only patients with active disease without glucocorticoid treatment were studied $(n=16)$ there was a significant correlation between neutrophil clustering activity and the number of neutrophils $\left(r_{s}=-0.64, p<0.05\right)$ (fig 5) and the total number of leucocytes $\left(r_{s}=0.63, p<0.05\right)$.

\section{CIRCULATING IMMUNE COMPLEXES}

Twenty nine of the 30 patients with active SLE had increased amounts of $\mathrm{Clq}$ binding circulating immune complexes (median $875 \mu \mathrm{g} / \mathrm{ml}$, range $<50-2300)$. Patients with major flares had higher concentrations of complexes than those with minor flares $(p<0.05)$. A weak correlation was found between $\mathrm{Clq}$ binding assay and neutrophil clustering activity $\left(r_{s}=0.4\right.$, $\mathrm{p}<0.05$ ). The $\mathrm{Clq}$ binding assay correlated inversely with neutrophil and total leucocyte counts in patients not treated with glucocorticoids $\left(r_{s}=0.61, p<0.05\right.$ and $r_{s}=0.55, p<0.05$ respectively).

SEQUENTIAL ANALYSIS OF NEUTROPHIL CLUSTERING ACTIVITY

Figure 6 shows the sequential follow up of two patients with high neutrophil clustering activity.

\section{Discussion}

This paper describes a simple and reproducible method for the measurement of serum neutrophil clustering activity. High clustering activity was found in most patients with active SLE, whereas patients with RA had mostly normal values. A clear relation was found between high neutrophil clustering activity and low peripheral neutrophil count in patients without glucocorticoid treatment.

It is well known that the neutrophil plays a central part in immune mediated vascular damage, and its implication in the pathogenesis of CNS disease ${ }^{8}$ and pulmonary vasculitis ${ }^{14}$ in SLE has been suggested. Neutropenia is regularly observed in active SLE. Several mechanisms are considered to be operative, including increased peripheral destruction, increased margination, and decreased granulopoiesis. ${ }^{15}$ It is interesting to note that all patients with active SLE and low neutrophil counts in this study had abnormal NCA. We observed normal neutrophil clustering activity during neutropenia induced by cytostatic treatment in SLE. One of our patients developed leucopenia during azathioprine treatment (fig 6a). In this case other disease manifestations suggested that the leucopenia was related to active SLE. Neutrophil clustering activity was very high at the time and it may be worth investigating whether this activity can be of help in discriminating between cytostatic drug induced or SLE activity induced cytopenia. Although glucocorticoids may alter the peripheral neutrophil count, they appeared to have no effect on neutrophil clustering 
activity, possibly because measurement of this activity reflects effects of circulating serum factors present in active SLE.

Increased concentrations of circulating immune complexes are found in most patients with active SLE, ${ }^{11}$ and the correlation with neutrophil clustering activity in this study may be due to independent factors related to disease activity. However, both $\mathrm{Clq}$ binding assay and neutrophil clustering activity correlated inversely with the neutrophil count. Therefore, serum neutrophil clustering activity may at least partly be due to immune complexes binding to neutrophil granulocytes through $\mathrm{Fc}$ or complement receptors. Further studies are warranted.

Our results are in some ways similar to those of Abramson et al ${ }^{8}$ and Hashimoto et al, ${ }^{16}$ who observed increased serum neutrophil aggregating activity in SLE, but mostly normal activity in sera from patients with RA. Hashimoto et al also found that the ability of human sera to generate neutrophil aggregation, adhesion to endothelial cells, and $\mathrm{O}_{2}^{-}$release were closely correlated. The exact relation between adhesion to endothelial cells, or aggregation measured in an aggregometer, and the present findings is not clear. Cytochalasin B affects neutrophil activation in several different ways. It causes aggregation to occur before degranulation ${ }^{17}$ and ablates stimulus specific aggregation responses, ${ }^{6}$ making its use for increasing the aggregation response debatable, and casting doubt on the relation to in vivo situations. It is possible that the present assay measures aggregating serum factors other than those measured by assays using cytochalasin $\mathrm{B}$.

Abramson et $a l^{8}$ found a particularly high neutrophil aggregating activity in serum samples from patients with CNS lupus, which suggests a relation with CNS vasculitis. Two of three patients with the highest neutrophil clustering activity in our study had hemianopia as evidence of cerebrovascular disease, and the third patient also had some evidence of CNS lupus (fig 6a). As a group, however, patients with CNS manifestations did not differ from the remainder, a finding perhaps explained by the different pathogenetic mechanisms operating.

Leucergy - that is, leucocyte agglomeration in citrated whole blood-has been studied by Berliner et $a l^{7}{ }^{7}$ In contrast with the findings in our study, he found increased leucergy in many rheumatic diseases and a response that correlated with erythrocyte sedimentation rate and disease activity. Different clustering/aggregating factors, perhaps related to the presence of platelets, may be operative in the leucergy test.

In conclusion, this simple and reproducible assay for neutrophil clustering in human sera may be a valuable tool in the study of human neutrophil behaviour. Its main drawback is that the cell separation and cell counting processes are rather time consuming. More knowledge about the predictive and diagnostic value of the test is necessary to determine its future clinical use.

We gratefully thank Gertrud Hellmer for skilful laboratory assistance. This investigation was supported by grants from Alfred Österlunds Stiftelse, Greta och Johan Kocks Stiftelser, Svenska livförsäkringsbolags nämnd för medicinsk forskning, Konung Gustaf V:s 80-årsfond, Thorsten och Elsa Segerfalks Stiftelse, Lunds Siukvo̊rdsdistrikt, Stiftelsen Bistånd åt Vanföra i Skane, The Medical Faculty of the University of Lund Crafoordska Stiftelsen, and Förenade Liv. Helgi Jonsson is recipient of the Syntex rheumatology prize 1988.

1 Hammerschmidt D, Weaver L, Hudson L, Craddock P R, Jacob H S. Association of complement activation and elevated plasma C5a with adult respiratory distress syndrome. Lancet 1980; i: 947-9.

2 Craddock P R, Hammerschmidt D, White J G, Dalmasso A P, Jacob H S. Complement (C5a)-induced granulocyte aggregation in vitro: a possible mechanism of complement mediated leukostasis and leukopenia. $\mathcal{F}$ Clin Invest 1977; 60: $260-4$.

3 Chenoweth D E, Cooper S W, Hugli T E, Stewart R W, Blackstone E H, Kirklin J W. Complement activation during cardiopulmonary bypass: evidence for generation of C3a and C5a anaphylotoxins. $N$ Engl f Medicine 1981; 304: C3a and $\mathrm{C}$.52.

4 Till G O, Ward P A. Lung injury secondary to chemotactic factor-induced leukocyte activation. Agents Actions 1983; 12 (suppl): 383-96.

5 Jacob H S. Neutrophil activation as a mechanism of injury. Semin Arthritis Rheum 1983; 13 (suppl 1): 144-7.

6 Ringertz B. Neutrophil aggregation-tactors modulating stimulus-specific responses. Acta Pathol Microbiol Immunol Scand [C] 1986; 94: 1-9.

7 Berliner S, Abramovitz M, Kariv N, et al. The leukergy test in rheumatic diseases: new implications for an old test. Arthritis Rheum 1985; 28: 899-903.

8 Abramson S B, Given W P, Edelson H S, Weissman G. Neutrophil aggregation induced by sera from patients with Neutrophil aggregation induced by sera from patients with 26: $630-6$.

9 Svensson B, Sturfelt G. Monocyte in vitro function in systemic lupus erythematosus (SLE). Glass adherence and spreading in the presence of SLE sera. Scand $\mathcal{F}$ Rheumato [Suppl] 1980; 31: 43-52.

10 Tan E M, Cohen A S, Fries J F, et al. The 1982 revised criteria for the classification of systemic lupus erythematosus. Arthritis Rheum 1982; 25: 1271-7.

11 Sturfelt G, Siöholm A G. Complement components, complement activation, and acute phase response in systemic lupus erythematosus. Int Arch Allergy Appl Immunol 1984; 75: 75-83.

12 Arnett F C, Edworthy S M, Bloch D A, et al. The American Rheumatism Association 1987 revised criteria for the Rheumatism Association 1987 revised criteria for the classification

13 Zubler R H, Lange G, Lambert P H, Miescher P A. Detection of immune complexes in unheated sera by a modified ${ }^{125} \mathrm{I}-\mathrm{Clq}$ binding test: effect of heating on the binding of $\mathrm{Clq}$ by immune complexes and applications of the test to systemic lupus erythematosus. F Immunol 1976 116: $232-5$.

14 Churg A, Franklin W, Chan K L, Kopp E, Carrington C B. Pulmonary hemorrhage and immune-complex deposition in the lung: complications in a patient with systemic lupus the lung: complications in a patient with systemic lup

15 Yamasaki K, Niho Y, Yanase T. Granulopoiesis in systemic lupus erythematosus. Arthritis Rheum 1983; 26: 516-21.

16 Hashimoto $Y$, Ziff $M$, Hued E. Increased endothelial cell adherence, aggregation and superoxide generation by neutrophils incubated in systemic lupus erythematosus and Felty's syndrome sera. Arthritis Rheum 1982; 25: 1409-18.

17 Kaplan H B, Edelson H S, Friedman R, Weissmann G. The roles of degranulation and superoxide anion generation in neutrophil aggregation. Biochim Biophys Acta 1982; 721 $55-63$. 